\title{
CO in Early Type Galaxies
}

\author{
Tommy Wiklind ${ }^{1}$ and Christian Henkel ${ }^{2}$
}

\author{
1. Onsala Space Observatory, S-43900 Onsala, Sweden \\ 2. Max-Planck-Institut für Radioastronomie, Auf dem Hügel 69, D-5900 Bonn, FRG
}

During the last two years, observations of the molecular cloud content of early type galaxies have drastically changed our view of these systems as inert gaspoor galaxies with little or no star formation activity. Systematic surveys in the $\mathrm{CO}(\mathrm{J}=1-0)$ line (Wiklind and Henkel, 1988a,b; Thronson, private communication) have shown that an IR selected sample of early type galaxies contains typically $10^{7}-10^{8} \mathrm{M}_{\circ}$ of molecular hydrogen gas. This is similar to the typical HI masses found in these galaxies (Knapp et al., 1985; Wardle and Knapp, 1986).

The majority of the detections comes from a survey that we are conducting, using the IRAM 30-m telescope and the newly constructed SEST telescope in Chile. We have also incoporated results from Thronson (private communication), as well as NGC 185 (Wiklind and Rydbeck, 1986) and NGC 4472 (Huchtmeier et al., 1988). The $\mathrm{H}_{2}$ masses have been computed from the $\mathrm{CO}$ integrated intensity, using a $\mathrm{N}\left(\mathrm{H}_{2}\right)$ / $\mathrm{I}_{\mathrm{CO}}$ conversion ratio of $2 \cdot 10^{20} \mathrm{~cm}^{-2}\left(\mathrm{~K} \mathrm{~km} \mathrm{~s}^{-1}\right)^{-1}$. The distances have been derived from our measurements of the radial velocities, corrected for the Solar motion relative to the center of the Local Group and for the Virgocentric flow (Aaronson et al., 1982). We have adopted a Hubble constant of $100 \mathrm{~km} \mathrm{~s}^{-1} \mathrm{Mpc}^{-1}$, with an assumed distance of $13.5 \mathrm{Mpc}$ to the Virgo cluster. Since both the conversion ratio and the assumed Hubble constant are "conservative", this means that the derived $\mathrm{H}_{2}$ masses are lower limits, unless the molecular cloud properties of the early type galaxies are greatly different from those of the Milky Way. For comparision with the molecular cloud properties of our sample of early type galaxics, we have from the litcraturc compiled a list of the molccular cloud propcrlies, as well as FIR and blue luminosities, for 123 spiral galaxies. In Figure 1 we show the $\mathrm{H}_{2}$ mass distributions for the two samples (light bars for the spirals, dark bars for the early type galaxies). It is evident that the spiral sample, on the average, has about an order of magnitude more $\mathrm{H}_{2}$ gas than the early type galaxies. A similar difference can be seen in Figure 2, which shows the distributions of the $\log (\mathrm{SFR})$. The star formation rate (SFR) can be derived directly from the FIR emission, as estimated by the IRAS, in a similar manner as Thronson and Telesco (1986). The SFR is here assumed to be directly proportional to the FIR luminosity: $\mathrm{SFR}=3.2 \cdot 10^{-10} \mathrm{~L}_{\mathrm{o}} \mathrm{M}_{\mathrm{o}} \mathrm{yr}^{-1}$. The typical star formation rates for the early type galaxies are around $0.1-1 \mathrm{M}$ $\mathrm{yr}^{-1}$. In Figure 3 we have plotted the distributions of the ratio of $\mathrm{F}_{60} / \mathrm{F}_{100}$, as measured by the IRAS. Although the $\mathrm{L}_{\mathrm{FR}}$ (SFR) distributions are different by about an order of magnitude, the distributions of the dust temperatures appear to be the same. Since we do not belive that the dust properties are significantly different in the early type galaxies than in spiral galaxies, this result means that the heating mechanism of the dust is as efficient in the early type sample as in the spiral sample. This indicates that the efficiency of star formation in early type galaxies might be higher than for the spiral sample. In Figure 4 we have plotted the distributions of $\log \left(\mathrm{L}_{\mathrm{RR}} / \mathrm{L}_{\mathrm{co}}\right)$ for the two samples. The ratio of $\mathrm{L}_{\mathbb{1 R}} / \mathrm{L}_{\mathrm{co}}$, which is proportional to the ratio $\mathrm{SFR} / \mathrm{M}\left(\mathrm{H}_{2}\right)$, is usually taken as a measure of the star formation efficiency (SFE). From this figure it is evident that the SFE for the early type galaxies is, on the average, as high or possibly higher than that of the spiral galaxies. This result may indicatc that spiral density waves are not necessary for efficient formation of massive stars. 
Acknowledgement. We are grateful to $\mathrm{Dr}$. H. Thronson for communicating results prior to publication.

\section{References:}

Aaronson, M., Huchra, J., Schechter, P.L. and Tully, R.B., 1982, Astrophys. J., 258, 64

Huchtmeier, W.K., Bregman, J.N., Hogg, D.E. and Roberts, M.S., 1988, Astron. Astrophys., 198, L17

Knapp, G.R., Turner, E.L. and Cunniffe, P.E., 1985, Astron. J., 90, 454

Thronson, H.A. and Telesco, C.M., 1986, Astrophys. J., 311, 98

Wardle, M. and Knapp, G.R., 1986, Astron. J., 91, 23 Wiklind, T. and Rydbeck, G., 1986, Astron. Astrophys., 164, L22

Wiklind, T. and Henkel, C., 1988a, submitted to Astron. Astrophys.

Wiklind, T. and Henkel, C., 1988b, in preparation

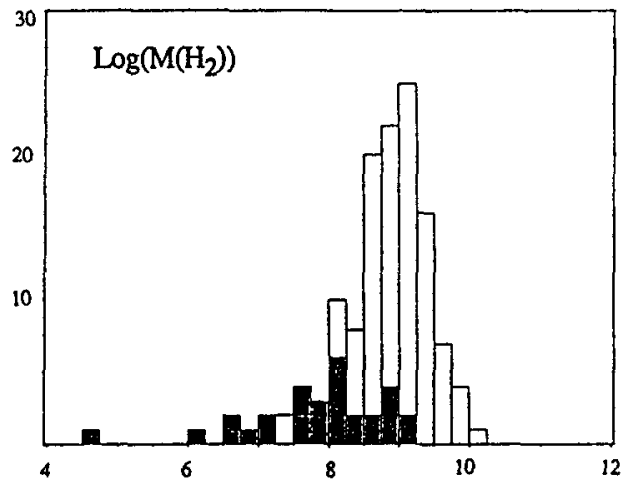

Figure 1

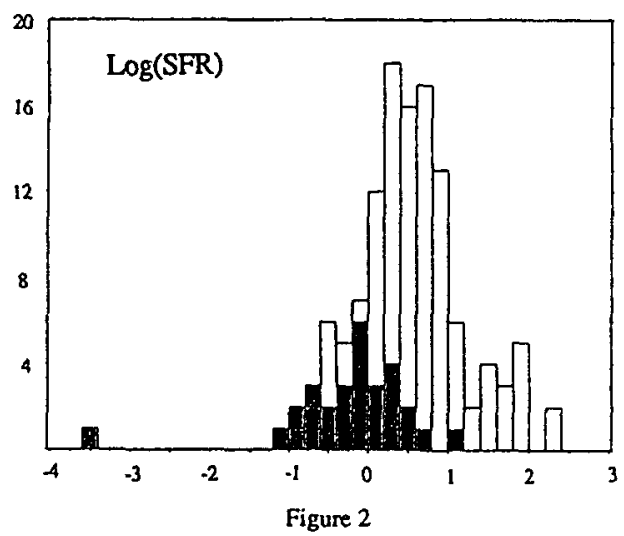

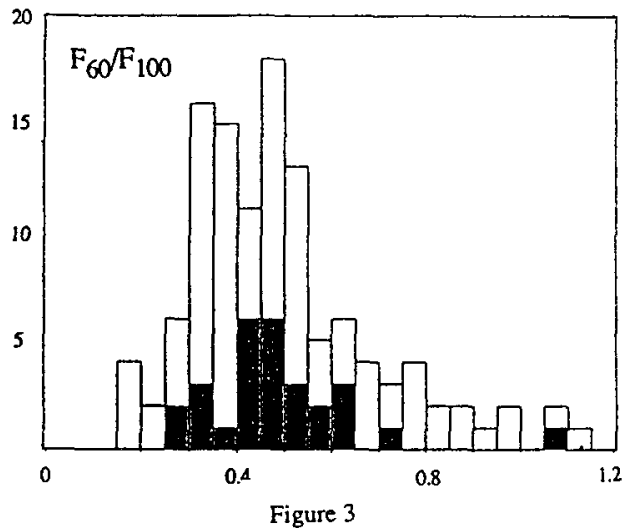

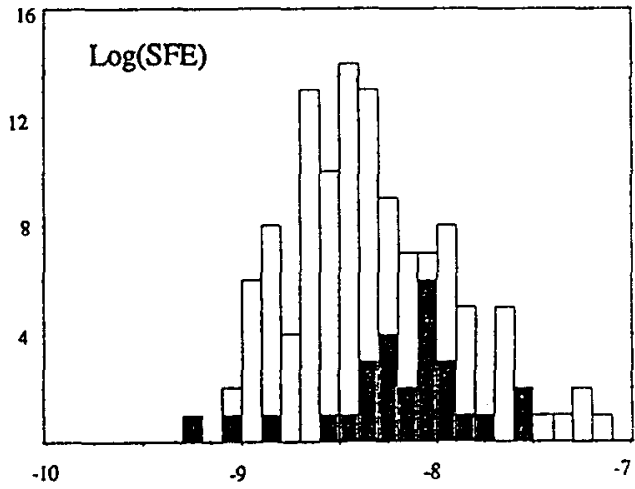

Figure 4 